\title{
Vehicle response on kinematic excitation
}

\author{
Veronika Valašková ${ }^{1,{ }^{*}}$, and Jozef Melcer ${ }^{1}$ \\ ${ }^{1}$ University of Zilina, Faculty of Civil Engineering, Department of Structural Mechanics and Applied \\ Mathematics, Univerzitna Street 8215/1, 01026 Zilina, Slovak Republic
}

\begin{abstract}
The vehicle - roadway interaction is actual engineering problem solved on many workplaces in the world. At the present time preference is given to numerical and experimental approaches. Vehicle designers are interested in the vibration of the vehicle and the forces acting on the vehicle. Civil engineers are interested in the load acting on the road. Solution of the problem can be carried out in time or in frequency domain. Road unevenness is the main source of kinematic excitation of the vehicle and therefore the main source of dynamic forces acting both on the road and the vehicle. The offered article deals with one of the possibilities of numerical analysis of the vehicle response in frequency domain. It works with quarter model of the vehicle. For the selected computational model of the vehicle it quantifies the Frequency Response Functions (FRF) of both force and kinematic quantities. It considers the stochastic road profile. The Power Spectral Density (PSD) of the road profile is used as input value for the calculation of Power Spectral Density of the response. All calculations are carried out numerically in the environment of program system MATLAB. When we know the modules of FRF or the Power Response Factors (PRF) of vehicle model the calculation of vehicle response in frequency domain is fast and efficient.
\end{abstract}

\section{Introduction}

The problem of rough surface road profiles and its influence on vehicle unwanted vibrations due to kinematic excitations is still a subject of research among automotive manufacturers and research groups, whose objective is to minimize their effects on the driver and passengers. Unevenness on the surface of the pavement is the main source of kinematic excitation of the vehicle. The real load acting on the roads is variable in time and in frequency composition. This should be known for the solution of many engineering tasks as lifetime, design, reliability, structure development, micro tremor, etc [1-3]. This paper presents simplest way how to obtain the Power Spectral Density (PSD) of input signal (in our case it is PSD of road unevenness), how to calculate the dynamic properties of analysed system (specifically the Power Response Factors) and PSD of output signals of individual quantities.

\footnotetext{
*Corresponding author: veronika.valaskova@,fstav.uniza.sk
} 


\section{Computational model of the vehicle}

The discrete (lumped mass) computational models of vehicles can be created on three qualitatively different levels: 1D - quarter model, 2D - plane model and 3D - spatial model. Every model has its advantages and disadvantages and under certain assumptions, it can be used for the solution of real practical problems. Quarter model of the vehicle is shown in Fig. 1. It can be used to model the effects of half of one axle of the vehicle on a traffic road and under certain simplifying assumptions to model the effects of the entire vehicle. The disadvantage of this model is that it can only model the heave effect of the upper mass on the contact forces between the wheel and the runway. It cannot model the pitch effect not the roll effect of the suspended mass on the contact force between the wheel and the runway.

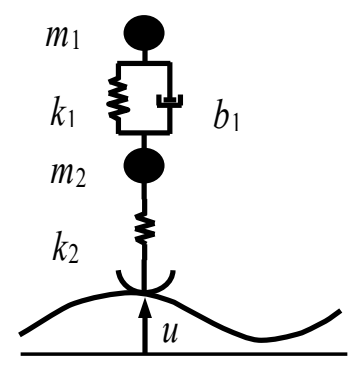

Fig. 1. Quarter model of the vehicle.

The behavior of the discrete computational model is described by the system of ordinary differential equations.

$$
\begin{gathered}
\ddot{r}_{1}(t)=\left\{-k_{1} \cdot\left[r_{1}(t)-r_{2}(t)\right]-b_{1} \cdot\left[\dot{r}_{1}(t)-\dot{r}_{2}(t)\right]\right\} / m_{1} \\
\ddot{r}_{2}(t)=\left\{+k_{1} \cdot\left[r_{1}(t)-r_{2}(t)\right]-k_{2} \cdot\left[r_{2}(t)-u(t)\right]+b_{1} \cdot\left[\dot{r}_{1}(t)-\dot{r}_{2}(t)\right]\right\} / m_{2}
\end{gathered}
$$

The contact forces are described by equation

$$
F(t)=F_{s}(t)+F_{d}(t)=-G+k_{2} \cdot\left[r_{2}(t)-u(t)\right] .
$$

The function $r_{1}(t)$ describes the time course of the vertical deflections of the upper mass $m_{1}$, the function $r_{2}(t)$ describes the time course of the vertical deflections of the lower mass $m_{2}$ and the function $u(t)$ describes the time course of the road profile. The stiffness and damping constants are denoted as $k_{1}, k_{2}, b_{1}$. [4].

\section{Frequency response function}

Frequency response function $F R(p)$, where $p=\mathrm{i} . \omega$ is a complex number, is defined as a ratio of steady-state response and harmonic excitation. If the excitation is harmonic with unit amplitude, then

$$
F R(p)=F R(\mathrm{i} \cdot \omega)=r_{\text {sts }}(t) /\left(h \cdot \mathrm{e}^{\mathrm{i} \cdot \omega \cdot t}\right)=r_{\text {sts }}(t) /\left(1 \cdot \mathrm{e}^{\mathrm{i} \cdot \omega \cdot t}\right)=r_{\text {sts }}(t) \cdot \mathrm{e}^{-\mathrm{i} \cdot \omega \cdot t} .
$$

Frequency response function $F R(p)$ as the function of complex variable can be expressed as

$$
F R(p)=|F R(p)| \cdot \mathrm{e}^{\mathrm{i} \cdot \phi},
$$


where $|F R(p)|$ is the absolute value or the magnitude of the frequency response. We can express $r_{\mathrm{sts}}(t)$ from equation (3)

$$
F R(p)=r_{\mathrm{sts}}(t) /\left(1 \cdot \mathrm{e}^{\mathrm{i} \cdot \omega \cdot t}\right) \quad \rightarrow \quad r_{\mathrm{sts}}(t)=F R(p) \cdot \mathrm{e}^{\mathrm{i} \cdot \omega \cdot t}
$$

After substituting (4) to (5)

$$
r_{\text {sts }}(t)=|F R(p)| \cdot \mathrm{e}^{\mathrm{i} \cdot \phi} \cdot \mathrm{e}^{\mathrm{i} \cdot \omega t}=|F R(p)| \cdot \mathrm{e}^{\mathrm{i} \cdot(\omega t+\phi)} .
$$

The graphic representation of frequency response is the frequency characteristic. The graphic representation of the absolute value (modulus) of frequency response is amplitude characteristic. The phase characteristic is the graphic representation of argument (phase) of frequency response in dependence on the frequency of harmonic excitation. The function $|F R(p)|^{2}$ is called power response factor (PRF).

The Laplace integral transform for the passing from time to frequency domain can be used. The Laplace image of some function $r(t)$ will be denoted as $L\{r(t)\}=R(p)$. In this case $p=\mathrm{i} \omega$ is the complex number. The function $r(t)$ and their derivatives with respect of time will be transformed as follows

$$
L\left\{r^{(n)}(t)\right\}=p^{n} \cdot R(p)-\sum_{i=1}^{n-1} p^{n-1-i} \cdot r^{(i)}(0+) \quad \text { for } n=1,2, \ldots \ldots
$$

The time functions $r_{1}(t), r_{2}(t)$ and $u(t)$ will be transformed on $R_{1}(p), R_{2}(p)$ and $U(p)$. The following designation can define two frequency responses

$$
\bar{r}_{i}=\frac{R_{i}(p)}{U(p)}, i=1,2
$$

By the Laplace transform of equations of motion and by defining the frequency responses we obtain the system of two equations in complex form for the function $\bar{r}_{i}(i=1,2)$. The matrix formulation is as follows

$$
[\mathrm{a}] \cdot\{\overline{\mathrm{r}}\}=\{\mathrm{RS}\} .
$$

Generally

$$
a_{i k}=a_{i k, \mathrm{re}}+\mathrm{i} \cdot a_{i k, \mathrm{im}}, \quad \bar{r}_{i}=\bar{r}_{i, \mathrm{re}}+\mathrm{i} \cdot \bar{r}_{i, \mathrm{~m}}, R S_{i}=R S_{i, \mathrm{re}}+\mathrm{i} \cdot R S_{i, \mathrm{im}} .
$$

Similarly, the frequency response of a dynamic component of contact force is defined as

$$
\bar{F}_{d}=\frac{L\left\{F_{d}(t)\right\}}{U(p)}=k_{2} \cdot\left(\bar{r}_{2}-1\right) .
$$

The Power Response Factors (PRF) of selected frequency functions at the speed of vehicle motion $10 \mathrm{~m} / \mathrm{s}$ are plotted on the following figures. The values of natural frequencies of the vehicle (calculated without damping) are introduced for better orientation: $f_{(1)}=1.06 \mathrm{~Hz}, f_{(2)}$ $=8.89 \mathrm{~Hz}$.

The frequency spectrum of the output signal can be obtained by multiplying the frequency response function of the system by the frequency spectrum of the input signal

$$
F S_{r}(p)=F R(p) \cdot F S_{u}(p) .
$$


By introducing the power spectral densities of the input signal $P S D_{u}(\omega)$ and output signal $P S D_{r}(\omega)$ it can be written

$$
\operatorname{PSD}_{r}(\omega)=|F R(p)|^{2} \cdot \operatorname{PSD}_{u}(\omega), \text { or }|F P(p)|^{2}=V S H_{r}(\omega) / V S H_{h}(\omega) .
$$
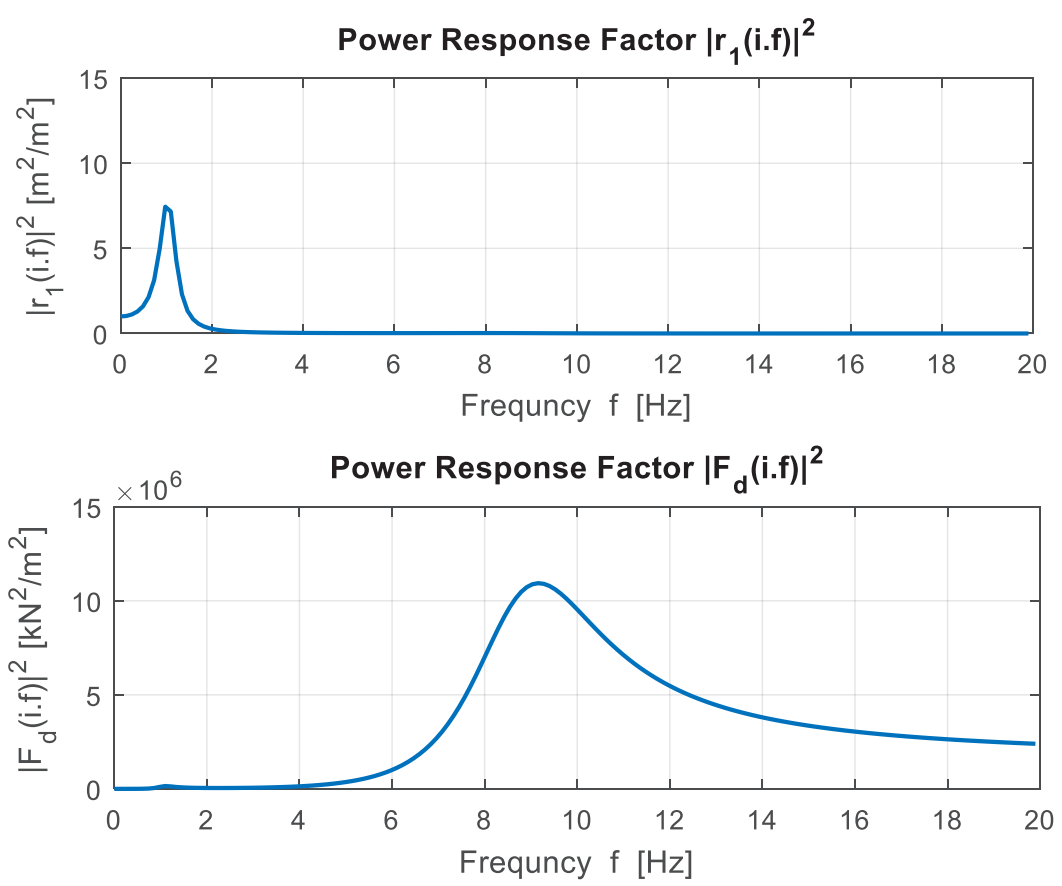

Fig. 2. Power response factor of selected frequency functions at the vehicle.

\section{Computational model of the road}

Assume the computational model of the road as rigid pavement with stochastic road profile. The stochastic road profile is shown in Fig. 3, it represents the source of kinematic excitation of the vehicle. The Power Spectral Density of the road profile is shown in Fig. 4.

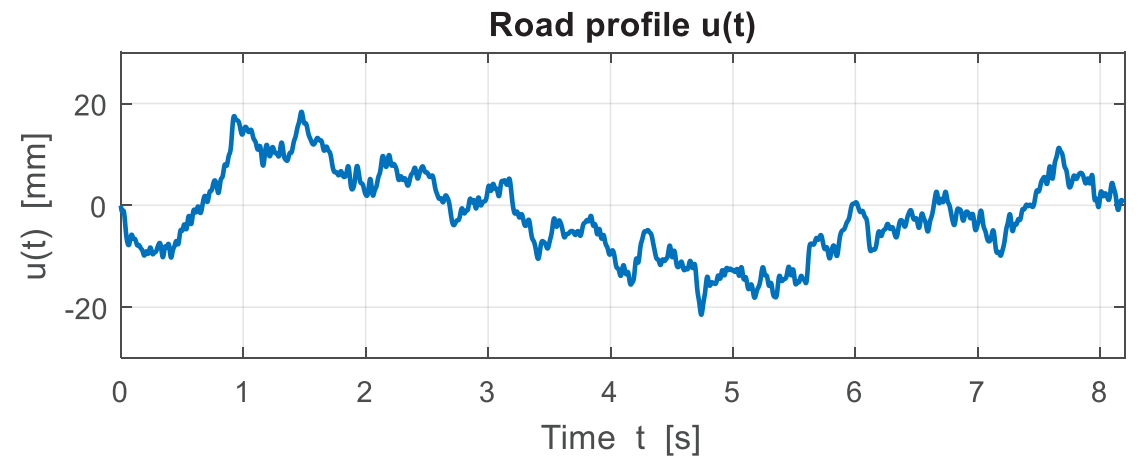

Fig. 3. The stochastic road profile. 


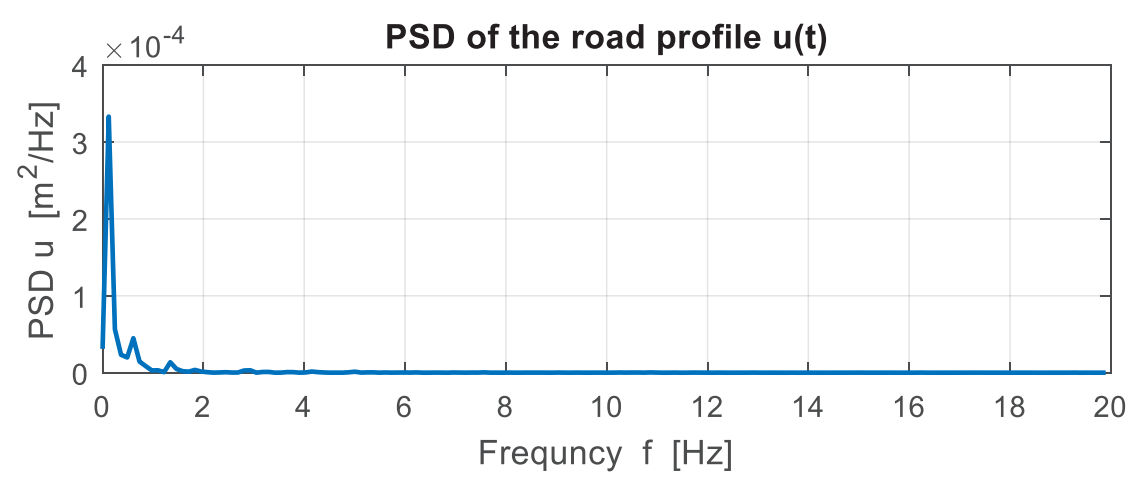

Fig. 4. Power Spectral Density.

\section{Solution of the dynamic response in frequency domain}

Solution of the dynamic response in frequency domain can be carried out in many ways. The simplest way is to obtain the Power Spectral Density of input signal, in our case the Power Spectral Density of road unevenness. Then to calculate the Frequency Response of analysed dynamical system specifically the Power Response Factors of individual quantities. And simply multiply the Power Response Factors of individual quantities by the Power Spectral Density of input signal, in our case by the Power Spectral Density of the road profile. The results thus obtained are shown in Fig. 5.
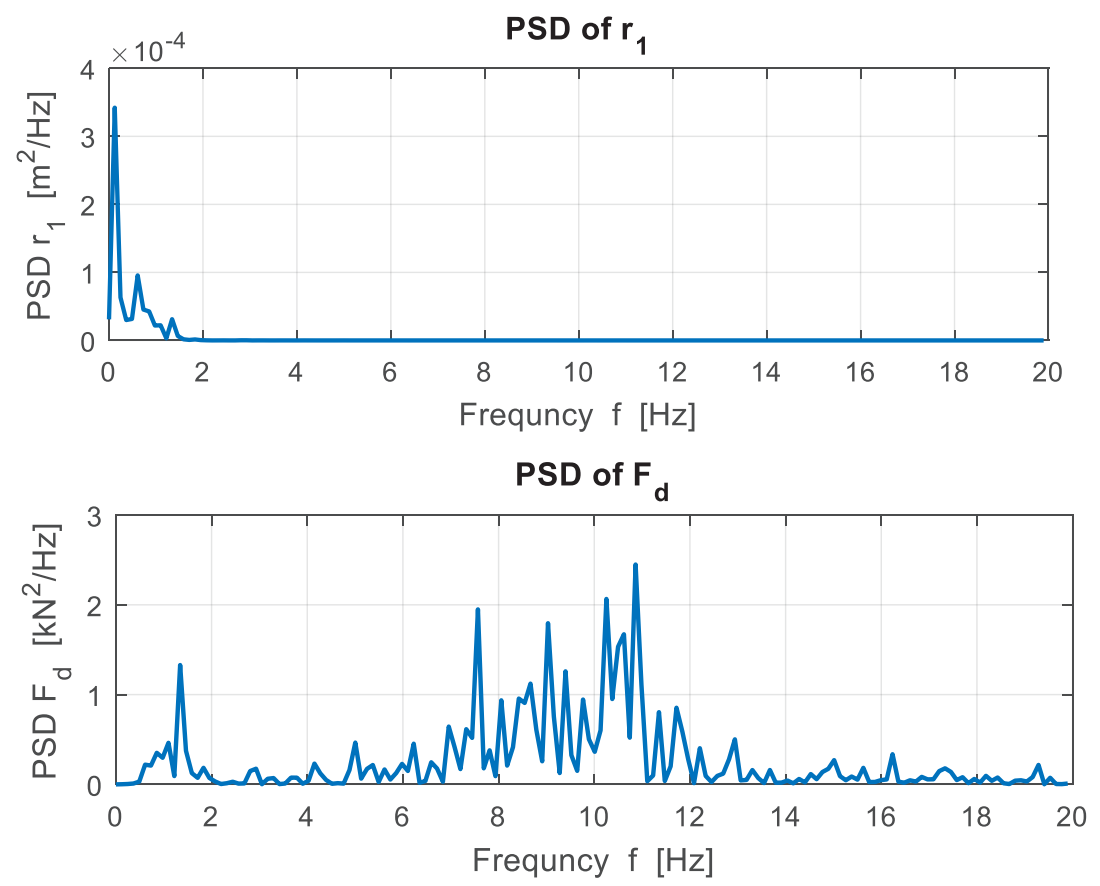

Fig. 5. Power Spectral Density of the road profile. 


\section{Conclusion}

Vehicle vibration induced by its motion along stochastic road profile can be solved in time or in the frequency domain. When we know the modules of FRF or the Power Response Factors (PRF) of the vehicle model, the calculation of vehicle response in the frequency domain is fast and efficient. In PSD of road profile predominate low frequencies (long waves). Upper mass $m_{1}$ sensitively responds to these low frequencies. The dynamic component of the contact force $F_{d}$ sensitively responds to frequencies close to the second natural frequency of the vehicle computational model, in frequency interval approximately from 7 to $11 \mathrm{~Hz}$.

This paper was supported by the Grant National Agency VEGA of the Slovak Republic (grant No. $1 / 0005 / 16)$.

\section{References}

[1] G. Verros, S. Natsiavas, G. Stepan, J Vib Control, 6, 1045-1063 (2000)

[2] M. Gobbi, G. Mastinu, J Sound Vib, 245(3), 457-481 (2001)

[3] S. Turkay, H. Akcay, J Sound Vib, 282(1-2), 111-124 (2005)

[4] J. Melcer, G. Lajčáková, I. Martinická, J. Králik, Dynamics of transport structures, EDIS, (2016) 